\title{
Integrative modeling to characterize structure and dynamics of biomolecules
}

\section{Florence Tama}

\author{
Nagoya University \& RIKEN, Nagoya, Japan
}

Hybrid and integrative modeling methods that combine computational molecular mechanics simulations with experimental data are powerful in describing the structure and dynamics of large biomolecules. In particular, flexible fitting is a powerful technique to build the 3D structures of biomolecules from cryo-electron microscopy (cryo-EM) density maps. While flexible fitting methods work nicely with very high-resolution maps, there are limitations for medium resolution maps ( $\sim 5-10$ angstrom $)$ in the case of complex conformational transitions. To overcome such issues, we proposed a refinement based on conformational ensemble, i.e., performing multiple fittings trials using various parameters. An automatic adjustment of the biasing force constants during the fitting process was introduced via a replica-exchange scheme to improve the success rate. From such multiple fittings, clustering analysis of the models obtained can be an effective approach to avoid over-fitting. In addition, we have looked into the pixel size parameter as it can impact the resolution and accuracy of a cryo-EM map, and we proposed a computational protocol to estimate the appropriate pixel size parameter. In our protocol, we fit and refine atomic structures against cryo-EM maps at multiple pixel sizes. The resulting fitted and refined structures are evaluated using the GOAP score. We have demonstrated the efficacy of this protocol in retrieving appropriate pixel sizes via several examples.

Keywords: cryo-EM, flexible fitting, dynamics, biomolecules 